\title{
La ictiofauna del yacimiento tartésico de la calle del Puerto, número 10 (Huelva): consideraciones generales
}

\author{
Eufrasia Roselló izouierdo y Arturo Morales Muñiz *
}

\section{INTRODUCCIÓN}

La ictiofauna del yacimiento tartésico de la calle del Puerto, número 10, está siendo objeto en estos momentos de un estudio pormenorizado que verá la luz en breve (Roselló y Morales, en prep.). Debido al interés que tanto cultural como ambientalmente reviste este sector de la fauna arqueológica consideramos oportuno realizar un avance de dicho informe en donde se comenta la importancia e implicaciones de estos hallazgos. Éste, a su vez, conlleva implicaciones adicionales sobre la actividad pesquera en el litoral sudoccidental durante el primer milenio a.C. que puede ser de importancia capital a la hora de elaborar teorias sobre la economía de las culturas locales.

\section{MATERIAL Y MÉTODOS}

Los restos analizados proceder de la excavación de urgencia realizada durante el verano de 1981 en el solar sito en el número 10 de la calle del Puerto (fig. 1) bajo la dirección del doctor J. P. Garrido Roiz.

- Laboratorio de Arqueozoología. Departamento de Biologia. Facultad de Ciencias. Universidad Autonoma de Madrid. Cantoblanco. 28049 Madrid. 
Estos materiales fueron recogidos a mano, no realizándose en momento alguno cribado o flotación de las muestras. En conjunto representan una pequeña colección de apenas un centenar de restos que están siendo analizados detalladamente en el Laboratorio de Arqueozoologia de la U.A.M. con ayuda de la osteoteca del Centro.

Aunque la metodología utilizada en este tipo de informes es con frecuencia diferente de la aplicada en análisis arquezoológicos más convencionales (por ejemplo, mamíferos o moluscos), la presente reseña tan solo pretende realizar un comentario de carácter general acerca de la importancia de esta fauna, por lo que se ha obviado cualquier referencia específica a técnicas de estudio, que pueden encontrarse en trabajos como los de Roselló (1989), Brinkhuizen (1989) o Wheeler \& Jones (1988).

\section{LA FAUNA}

Se han podido detectar en las muestras remitidas un total de 14 especies de peces, tres de ellas pertenecientes a los condrictios (peces cartilagionosos) y 11 de osteictios (peces óseos) de las cuales 10 lo son de teleósteos y una (el esturión, Acipenser sturio) de la infraclase de los condrósteos. Además de éstas, hay otras dos especies adicionales de condrictios identificadas tentativamente a especie (musola, Mustelus mustelus) y a género (Raja), respectivamente (Cuadro 1). Debemos señalar que dos especies constituyen primeras citas para la protohistoria española y, de éstas, una lo es además para la Península Ibérica.

Desde un punto de vista estrictamente faunístico llama la atención lo diversificado de la asociación recuperada. Aunque no podemos realizar afirmaciones cuantitativas fiables con una base de datos tan exigua sí podemos apreciar una riqueza que, desde luego, no corre pareja con el número de piezas recuperadas. El valor estimado de diversidad según la fórmula de Shannon confirma tal postulado $\left(H^{\prime}=3^{\prime} 41\right)$. Por otra parte, esta diversidad taxonómica lo es también ecológica dado que poseemos tanto especies costeras (espáridos) como neriticas o pelágicas (túnidos, grandes escualos) y, dentro de las costeras, tanto fauna bentónica (Raja sp., "rayas"; Mustelus aff. mustelus, musola; Muraena helena, morena) como nerítica (espáridos en general); tampoco faltan especies anfidromas (Acipenser sturio, esturión; ¿Sparus aurata?, dorada). Como suele ocurrir con casi todos los peces, los recuperados en calle del Puerto son fun- 


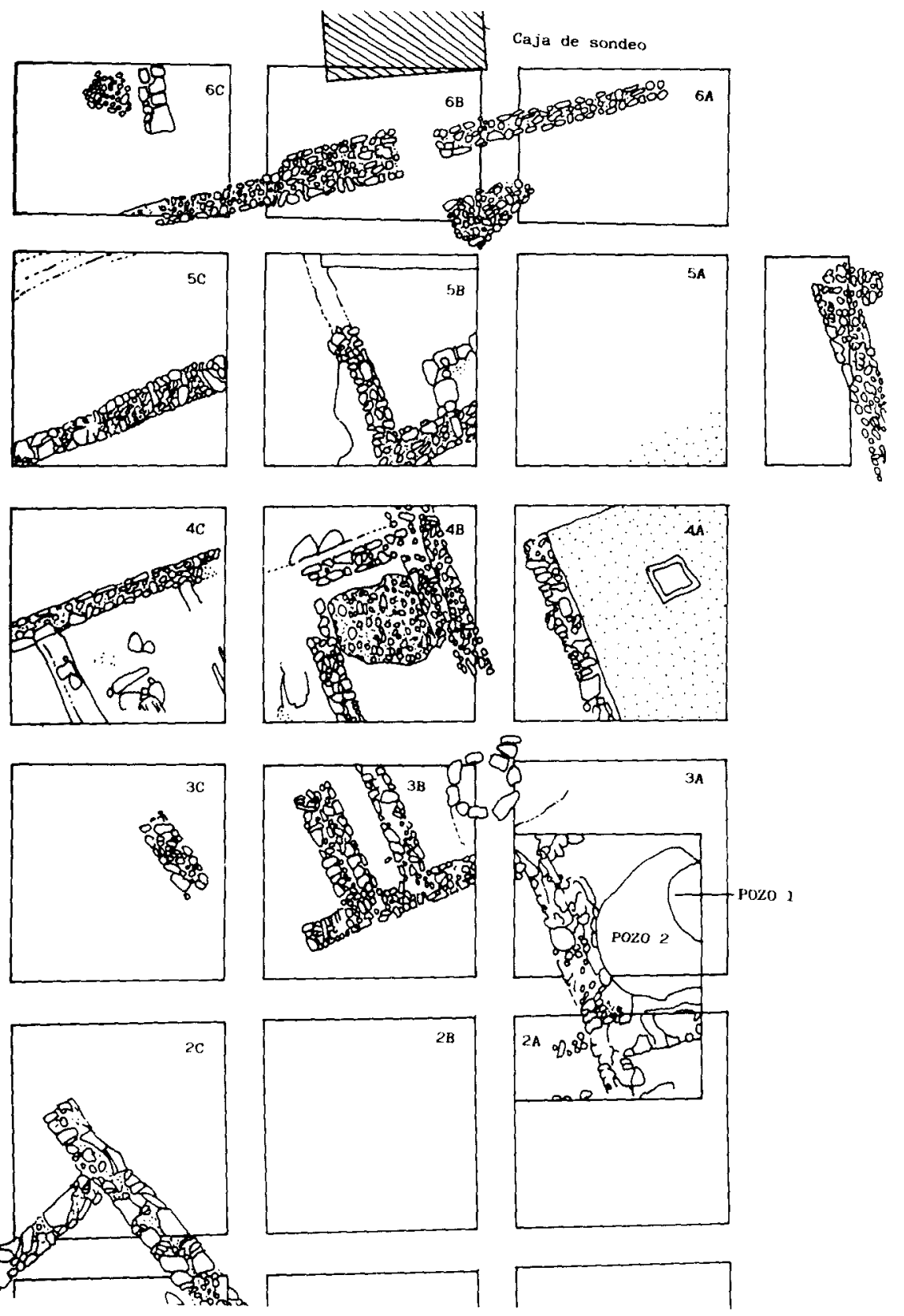

Fig. 1. Representación gráfica de la excavación de la calle del Puerto, $n .^{\circ} 10$. 
CLASE CHONDRICTHYES (peces cartilaginosos)

SUBCLASE ELASMOBRANQUIOS

ORDEN GALEIFORMES (escualos)

Familia ODONTASPIDIDOS

1. Odontaspis taursus, pez toro (1)

Familia LAMNIDOS

2. Isurus oxyrinchus, marrajo (1)

Familia TRIAKIDAE

3. Mustelus aff. mustelus, musola (9)

ORDEN RAYIFORMES (rayas)

Familia MILIOBATIDOS

4. Myliobatis aquila, águila marina (1) $\left({ }^{\circ}\right)$

Familia RAYIDOS

5. Raja sp., raya (1)

CLASE OSTEICHTHYES (peces oseos)

INFRACLASE CONDRÓSTEOS

Familia ACIPENSÉRIDOS (esturiones)

6. Acipenser sturio, esturión (3)

INFRACLASE TELEOSTEOS

ORDEN ANGUILIFORMES

Familia MURENIDOS

7. Muraena helena, morena (1)

ORDEN PERCIFORMES

Familia SERRANIDOS

8. Epinephelus guaza, mero (1)

Familia ESPÁRIDOS

9. Dentex dentex, dentón común (17)

10. Pagellus erythrinus, breca (2)

11. Pagrus caeruleostictus, zapata (3) (*)

12. Pagrus pagrus, pargo (5)

13. Sparus aurata, dorada (6)

Familia ESCIENIDOS

14. Argyrosomus regius, corvina (12)

Familia TÚNIDOS

15. Thunnus thynnus, atún (11)

Familia HEMÚLIDOS

16. Plectorhinchus mediterraneus, burro (6) $\left({ }^{\circ}\right)$

Cuadro 1. Relación de especies de peces identificadas en calle del Puerto, número 10. El orden de las familias de Perciformes es arbitrario. Los números entre paréntesis indican piezas pertenecientes a cada una de ellas. $(\%)=$ primera cita para la protohistoria española. (*) = primera cita para la protohistoria ibérica. 
damentalmente carnivoros aunque los grupos sobre los que depredan varian bastante. Llama la atención, por lo frecuentes que han sido las recuperaciones de conchas en el yacimiento, la presencia de algunas especies fundamental o estrictamente malacófagas (generos Pagellus, Pagrus y Sparus) que, como en el caso del águila de mar (Myliobatis aquila), son visitantes asiduos de los bancos de ostras.

Entre las especies que se alimentan de pequeños invertebrados (en general) mencionaremos el esturión (Acipenser sturio) y la corvina (Argyrosomus regius). Ictiófagos más estrictos serían el mero (Epinephelus guaza) y el atún rojo (Thunnus thynnus) asi como los grandes escualos quienes tampoco desechan la carroña. Éstos y el atún son grandes migradores por lo que su aparición en el yacimiento viene a indicarnos un tipo de pesca estacional que aún nos queda por precisar, aunque parece ser a todas luces primaveral.

Un último dato referido a los aspectos meramente biológicos de la asociación faunistica es el relativo a la abundancia específica. La ictiofauna está dominada por los espáridos, que son elementos comunes en estas costas actualmente. Les siguen en importancia corvinas, atunes y burros tampoco excepcionales en la zona hoy aunque cada vez vayan siendo más infrecuentes debido a la pesca intensiva de la que son objeto. El resto de las especies, muy poco abundantes en la muestra, aparecen también con frecuencia en nuestras costas. Es muy posible que este tipo de composición de la muestra se deba tanto a causas naturales (reflejo de la auténtica abundancia pretérita de aquellos ecosistemas) como a culturales sobre las que pasamos a realizar algunos comentarios a continuación.

El primer punto que llama poderosamente la atención sobre estas especies es su enorme tamaño. De momento sólo hemos podido inferir éste indirectamente comparando las muestras con los ejemplares de nuestras colecciones comparativas y extrapolando de un modo grosero. Aunque confiamos poder llevar a cabo en el futuro unas estimaciones fiables, basadas en el cáiculo de la regresión, adelantemos que prácticamente todos los restos de peces recuperados poseen tallas que, para cada una de las diferentes especies consideradas, se encuentran en valores próximos a los máximos constatados para dichas especies en la actualidad. En algunos casos (zapata, dentón) las tallas constatadas podrian considerarse hoy en día como excepcionales. Este aspecto parece ser debido a una recuperación parcial del resto y a la pérdida tafonómica que la misma implica. 
Esta última conclusión también se refuerza con el hecho de que no parece lógico postular una selectividad pesquera centrada en la exclusiva explotación de las mayores ejemplares de cada especie. En primer lugar, porque las especies recuperadas difieren mucho en sus tallas máximas $y$, en segundo lugar, porque se nos antoja impracticable un determinismo pesquero de esta indole. Hoy en día, por ejemplo, a pesar de toda la sofisticación pesquera sería absolutamente imposible tal práctica. La pesca parece ser más bien un fenómeno de tipo aleatorio determinado por unas leyes ciertamente complejas y que se resisten a los intentos de la cuantificación o formulación matemática.

A pesar de todo, no deja de ser interesante especular con el hecho de que tales tallas nos están evidenciando una etapa inicial de la predopresión humana sobre las poblaciones ícticas protohistóricas de aquel litoral onubense. En esos momentos la explotación del recurso no habria alcanzado aún las cotas de industrialización actuales y ello supondría una extracción no dañina para la dinámica de las poblaciones, que podrían continuar conservando individuos vivos durante un lapso temporal más grande. Esto acarrearía, para un patrón de crecimiento continuado como es el propio en peces, tallas que hoy en día, a causa de esta misma presión, son sencillamente imposibles de alcanzar. Esta hipótesis se ve reforzada con las investigaciones realizadas por nosotros en torno a la evolución de las tallas ocurridas desde el Neolítico en ictiofaunas de interés comercial de las costas andaluzas. Este trabajo ha sido objeto de un tratamiento detallado en un trabajo previo (Roselló, 1989) y, hasta cierto punto, contradice las conclusiones que en torno a las tallas acabamos de comentar. Evidentemente, sólo recuperaciones de fauna cribada pueden dar cumplida respuesta a estos interrogantes.

Un último punto sobre el que merece la pena mencionar se refiere al fenómeno de la tecnología pesquera. Las diferencias de hábitos ecológicos de las especies reconocidas en la calle del Puerto nos obligan a pensar obligatoriamente en una tecnología pesquera altamente sofisticada que incluiría no sólo arpones o anzuelos sino redes o artes de captura similares (¿trasmallos?, ¿liñas?, ¿palangres? e incluso ¿almadrabas?). De lo que no cabe duda ya es de una cosa: la riqueza íctica del litoral andaluz y, muy especialmente, del occidental, habría sido ya percibida desde mucho antes del Hierro por las poblaciones litorales andaluzas quienes, sin duda, supieron responder a la necesidad de explotarlas de un modo adecuado y rentable.

Estudios detallado sobre ictiofaunas como la recuperada en la calle del Puerto, número 10, pueden guardar la respuesta a muchos de los 
interrogantes que tenemos planteados en estos momentos los arqueozoólogos y arqueólogos españoles, ya que los peces representan un elemento básico en las economías de los asentamientos costeros de nuestro país desde tiempos prehistóricos. 


\section{BIBLIOGRAFIA}

BRInkHUIZEN, D., (1989): Ichthyo-archeologisch onderzoek: Methoden en toepassing aan de hand van romeins vismaterial uit Velsen (Nederland). Tesis Doctoral. Rijksuniversiteit Groningen.

Roselló, E., y Morales, A. (en prep.): Estudio arqueozoológico de la ictiofauna recuperada en la calle del Puerto, núm. 10 (Huelva).

Rosello, E., (1989): Arqueoictiofaunas ibéricas. Aproximación metodológica y bio-cultural. Tesis Doctoral. Universidad Autónoma de Madrid, 688 págs.

WHEELER, A., JONES, A. K., (1989): The study of fish remains from archaeological sites. Cambridge University Press. 